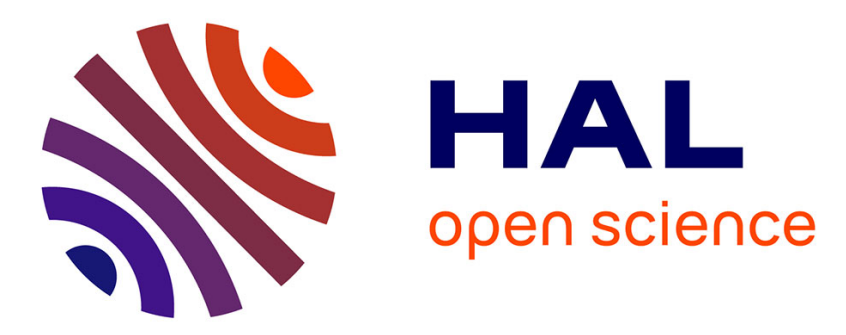

\title{
Pure and achromatic spin-orbit shaping of light from Fresnel reflection off space-variant anisotropic media
}

Mikaël Ghadimi Nassiri, Etienne Brasselet

\section{To cite this version:}

Mikaël Ghadimi Nassiri, Etienne Brasselet. Pure and achromatic spin-orbit shaping of light from Fresnel reflection off space-variant anisotropic media. Physical Review A : Atomic, molecular, and optical physics [1990-2015], 2019, 99 (1), pp.013836 (1-5). 10.1103/PhysRevA.99.013836 . hal-02104446

\section{HAL Id: hal-02104446 \\ https://hal.science/hal-02104446}

Submitted on 19 Apr 2019

HAL is a multi-disciplinary open access archive for the deposit and dissemination of scientific research documents, whether they are published or not. The documents may come from teaching and research institutions in France or abroad, or from public or private research centers.
L'archive ouverte pluridisciplinaire HAL, est destinée au dépôt et à la diffusion de documents scientifiques de niveau recherche, publiés ou non, émanant des établissements d'enseignement et de recherche français ou étrangers, des laboratoires publics ou privés. 


\title{
Pure and achromatic spin-orbit shaping of light from Fresnel reflection off space-variant anisotropic media
}

\author{
Mikaël Ghadimi Nassiri and Etienne Brasselet* \\ Université de Bordeaux, CNRS, LOMA, UMR 5798, F-33400 Talence, France
}

(Received 8 October 2018; published 22 January 2019)

\begin{abstract}
We propose an approach to achieve achromatic pure geometric phase shaping of light from anisotropic media by exploiting the fundamental laws of electromagnetic waves. Its practical implementation requires a cover layer made of a dispersive isotropic medium. The approach applies to any anisotropic material and any geometric phase spatial distribution and preserves the geometric phase reversal upon the reversal of the incident polarization handedness. An experimental demonstration is made over the whole visible range but can be extended to any wavelength range without conceptual issues.
\end{abstract}

DOI: 10.1103/PhysRevA.99.013836

\section{INTRODUCTION}

In wave physics, the fact that phase velocity depends on frequency, i.e., dispersion, is a generic phenomenon common to all waves. Dispersion is ubiquitous to wave-matter interactions and is mathematically formulated by the KramersKronig relations that link the real and imaginary parts of any holomorphic functions. An every day striking manifestation of chromatic dispersion pertaining to electromagnetic waves is the rainbow, which takes place as water droplets refract the different colors of sunlight into distinct directions. From a practical point of view, dispersion of optical waves is either an asset or a drawback. An example of the former situation can be found in spectroscopy where systems with large dispersion are sought to achieve high-resolution performances. In contrast, the dispersive nature of optical materials is detrimental for imaging using polychromatic illumination since each wavelength eventually produces a spatially distinct image. Of course, dispersion-management schemes have been developed to either amplify or attenuate its effects depending on the purpose.

In this context, the present work addresses the achromatization issue of two-dimensional flat optics made of spacevarying anisotropic media. The latter represent a class of flat-optics optical elements enabling spatial phase modulation by design according to an approach articulated by Bhandari two decades ago [1]. Such geometric phase optical elements allow imprinting the structural information associated with the in-plane spatial distribution of the optical axis orientation angle $\psi$ into the phase of a light field. Considering an incident circularly polarized beam, the process only affects the fraction of light that experiences reversal of the incident spin angular momentum along the incident propagation direction (which defines the $z$ axis in this work) as it interacts with the device. The corresponding component of the light field acquires an extra phase lag of a geometrical nature, $\Phi_{g}= \pm 2 \psi$, where the \pm sign depends on the handedness of the inci-

\footnotetext{
*etienne.brasselet@u-bordeaux.fr
}

dent circular polarization state. This practically means that the purity of the process, i.e., the power fraction $\eta$ of the emerging light acquiring the hard-coded geometric phase, is wavelength modulated. More precisely, assuming nonabsorbing optical materials, $\eta(\lambda)=\sin ^{2}[\pi \delta n(\lambda) L / \lambda]$, where $\delta n(\lambda)$ is the wavelength-dependent birefringence of the optical element and $L$ its thickness. Therefore, considering an incident polychromatic polarized light, the output field is $a$ priori a spectrally nonuniform superposition of processed and nonprocessed circularly polarized components. Even though a brute-force broadband polarization filtering may select the processed component in the case of incident circular polarization [2], purity dispersion prevents the full deployment in the spectral domain of what makes geometric phase optical elements attractive in both the classical and quantum regimes [3]. In particular, the generation of polychromatic optical states with nonseparable polarization and spatial degrees of freedom (a classical optics example being the so-called vector beams [4]) implies dispersion-free purity.

To tackle this issue, several approximate dispersioncompensation schemes have been proposed, independently of the origin of the birefringence, namely, artificial (case 1) or natural (case 2). Case 1 refers to form birefringence originating from subwavelength gratings [5], for which the grating can be designed to exhibit approximate anomalous birefringence dispersion of the form $\delta n(\lambda) \propto \lambda$, hence compensating the $1 / \lambda$ dependence of the purity. This was first discussed toward the elaboration of broadband birefringent optical retarders [6] and later experimentally implemented in the case of geometric phase polychromatic vectorial vortex beam generators in the midinfrared domain [7]. Practical applications of formbirefringence dispersion management have also emerged such as the development of high-contrast astronomical imaging instruments, for which broadband vectorial vortex phase masks are desirable [8]. In case 2 an effective dispersioncompensation scheme can also be obtained by design by breaking the translational invariance along the propagation direction of light. Here again, this was first discussed in the framework of broadband optical retarders $[9,10]$ obtained by stacking several uniform birefringent slabs. Liquid-crystal 
technology eventually allowed creating the continuous version of the latter idea via three-dimensional structuring of the optical axis. This led to the advent of liquid-crystal geometric phase optical elements with respectable trade-off between the spectral bandwidth, transmittance, and purity [11,12], which had remained under development until now $[13,14]$. Recently, the use of helix-based liquid crystals exhibiting the circular Bragg reflection phenomenon [15] was shown to provide broadband features without the need of artificial structuring along the propagation direction [16-19]. Still, all these approaches are approximate and designed to work on a finite spectral bandwidth.

Here we address the question whether it is possible to achieve achromatic pure geometric phase shaping of light using space-variant anisotropic media without relying on intrinsically approximate dispersion-compensation schemes as explored to date. We answer positively to this question by proposing an approach that finds its root in the rotational symmetry breaking at the interface between isotropic and anisotropic media.

\section{POSITION OF THE PROBLEM}

Let us consider a circularly polarized plane wave propagating along the $z$ axis impinging at normal incidence on a nonabsorbing uniaxial slab lying in the $(x, y)$ plane and whose optical axis is aligned along the $x$ axis. Using Jones notation in the reference frame $(\mathbf{x}, \mathbf{y})$, the incident $(i)$ and reflected $(r)$ waves are expressed, respectively, as

$$
\mathbf{E}_{i}=\frac{E_{0}}{\sqrt{2}}\left(\begin{array}{c}
1 \\
i \sigma
\end{array}\right), \quad \mathbf{E}_{r}=\frac{E_{0}}{\sqrt{2}}\left(\begin{array}{c}
r_{\|} \\
i \sigma r_{\perp}
\end{array}\right),
$$

where $E_{0}$ is the electric-field magnitude, $\sigma= \pm 1$ refers to the sense of rotation of the electric field in the plane $(x, y)$, and $r_{\|, \perp}$ are the Fresnel reflection coefficients for the electric-field components polarized parallel and perpendicular to the optical axis, respectively. The fraction $\xi$ of the reflected light with reversed projection of the spin angular momentum along the $z$ axis is therefore evaluated as

$$
\xi=\frac{\left|\mathbf{E}_{r} \cdot \mathbf{c}_{-\sigma}^{*}\right|^{2}}{\left|\mathbf{E}_{r}\right|^{2}} .
$$

where $\mathbf{c}_{\sigma}=(\mathbf{x}+i \sigma \mathbf{y}) / \sqrt{2}$ is the circular polarization unit vector. From Eqs. (1) and (2) one obtains

$$
\xi=\frac{1}{2} \frac{\left(r_{\|}-r_{\perp}\right)^{2}}{r_{\|}^{2}+r_{\perp}^{2}} .
$$

In the typical case of an interface between the vacuum and the uniaxial medium characterized by a birefringence 0.1 and an average refractive index 1.5 , Eq. (3) gives $\xi \sim 10^{-2}$. This corresponds to reflected light having a polarization ellipse almost identical to the incident one in the plane $(x, y)$. This picture, however, may drastically change when the vacuum is replaced by an isotropic medium with refractive index $n$. Indeed, recalling that $r_{\|}=\left(n-n_{\|}\right) /\left(n+n_{\|}\right)$and $r_{\perp}=\left(n-n_{\perp}\right) /$ $\left(n+n_{\perp}\right)$, where $n_{\|, \perp}$ are the refractive indices parallel and perpendicular to the optical axis, one gets, from Eq. (3),

$$
\xi=\left[1+\left(\frac{n^{2}-\bar{n}^{2}}{n \delta n}\right)^{2}\right]^{-1},
$$

where $\bar{n}=\left(n_{\|} n_{\perp}\right)^{1 / 2}$ and $\delta n=n_{\|}-n_{\perp}$. Therefore, $\xi=1$ when $n=\bar{n}$, which corresponds to $r_{\perp}=-r_{\|}=r^{*}$, irrespective of the birefringence value. That is to say, the sought achromatic half-wave birefringent phase retardation is obtained in the reflection mode.

Combining the approach above with spatial rotations of the anisotropic dielectric tensor allows shaping the phase of the reflected light based on geometric phase optical elements. Concretely, assuming a space-variant optical axis orientation angle $\psi(x, y)$ and applying the Jones formalism to describe the reflected field, one gets

$\mathbf{E}_{r}=\left(\begin{array}{cc}\cos \psi & -\sin \psi \\ \sin \psi & \cos \psi\end{array}\right)\left(\begin{array}{cc}r_{\|} & 0 \\ 0 & r_{\perp}\end{array}\right)\left(\begin{array}{cc}\cos \psi & \sin \psi \\ -\sin \psi & \cos \psi\end{array}\right) \mathbf{E}_{i}$,

which simplifies to

$$
\mathbf{E}_{r}=\frac{E_{0}}{2 \sqrt{2}}\left[\left(r_{\|}+r_{\perp}\right)\left(\begin{array}{c}
1 \\
i \sigma
\end{array}\right)+\left(r_{\|}-r_{\perp}\right) e^{2 i \sigma \psi}\left(\begin{array}{c}
1 \\
-i \sigma
\end{array}\right)\right] .
$$

One recognizes the geometric phase term $e^{i \Phi_{g}}$ in the second term of Eq. (6), which is associated with the fraction of reflected light with reversed spin angular momentum with respect to that of the incident field. The purity of the process $\eta$ equals $\xi$ [see Eq. (3)]. Therefore, it is possible to impart a geometric phase to the reflected light in a pure and achromatic manner provided that $r_{\|}+r_{\perp}=0$ whatever the wavelength, hence when the condition $n(\lambda)=\bar{n}(\lambda)$ is satisfied.

\section{EXPERIMENTS}

\section{A. Refractive index matching}

Experimentally, the above approach is first tested by using a calcite slab, $5 \mathrm{~mm}$ thick, coated by a nonabsorbing oil layer with refractive index $n$. A set of oils is chosen from the refractive index liquids catalog of Cargille Laboratories. The fraction $\xi$ is measured at $532 \mathrm{~nm}$ by using a continuouswave circularly polarized Gaussian laser beam with a waist diameter of $\sim 200 \mu \mathrm{m}$. In practice, the beam reflected off the oil/calcite interface is selected by sandwiching the oil layer between the calcite crystal and a slightly tilted glass coverslip. Then $\xi$ is evaluated by measuring the power of the two circularly polarized reflected components $P_{\text {preserved }}$ and $P_{\text {reversed }}$, which respectively refer to the power of the reflected light beam components having preserved and reversed spin angular momentum contributions, namely,

$$
\xi=\frac{P_{\text {reversed }}}{P_{\text {preserved }}+P_{\text {reversed }}} .
$$

Experimental measurements (Fig. 1, markers) are well described by the behavior predicted by Eq. (4) (Fig. 1, red curve), taking $n_{\|}=1.498$ and $n_{\perp}=1.663$ for the main refractive indices of the calcite at $\lambda=532 \mathrm{~nm}$. The latter values are evaluated from the refractive index data reported in Ref. [20] accounting for the fact that our calcite crystal has its optical axis oriented at an angle $15^{\circ}$ from the $(x, y)$ plane. The obtained maximum value $\xi_{\max } \simeq 1$ demonstrates that one obtains a reflected light having an almost pure degree of spin angular momentum reversal. The departure from the ideal unit 


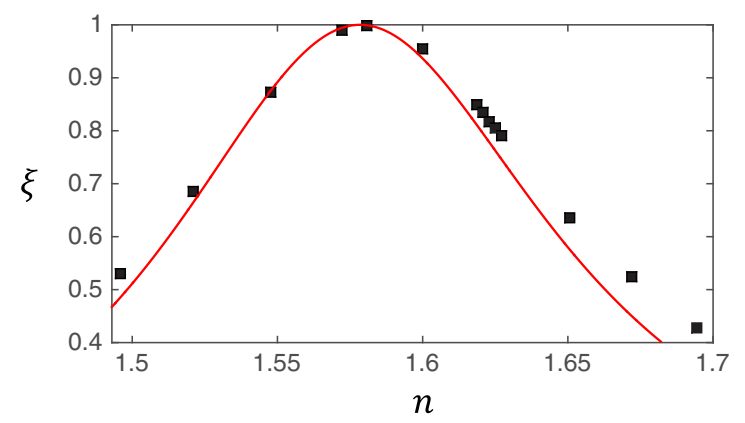

FIG. 1. Purity $\xi$ as a function of the refractive index $n$ of the isotropic cover layer. Markers show experimental data and the solid curve denotes theory [see Eq. (4)]. The values of $n$ at $532 \mathrm{~nm}$ are extrapolated from the known values at $\lambda_{\mathrm{F}}=486.1 \mathrm{~nm}, \lambda_{\mathrm{D}}=589.3 \mathrm{~nm}$, and $\lambda_{\mathrm{C}}=656.3 \mathrm{~nm}$ by using a Cauchy law of the form $n=c_{1}+$ $c_{2} / \lambda^{2}$.

value is of the order of $2 \times 10^{-3}$ and is explained by the finite precision of our circular polarizers.

\section{B. Achromatic behavior}

In the second step, the possibility to achieve dispersionfree pure reflective geometric phase shaping is experimentally addressed in the visible domain. This is done by using the oil that gives the maximal value for $\xi$ at 532-nm wavelength (see Fig. 1). The dispersion of the latter oil is shown in Fig. 2(a) (magenta curve), where the three crosses refer to the tabulated values at $\lambda_{\mathrm{F}}=486.1 \mathrm{~nm}, \lambda_{\mathrm{D}}=589.3 \mathrm{~nm}$, and $\lambda_{\mathrm{C}}=656.3 \mathrm{~nm}$ and the solid curve is the Cauchy extrapolation given by $n=$ $1.5453+0.010036 / \lambda^{2}$ (taking the wavelength in micrometer units). The departure from the sought dispersion behavior $n(\lambda)=\bar{n}(\lambda)$ (black curve) can be visually appreciated in Fig. 2(a) and corresponds to a standard deviation $\simeq 6 \times 10^{-3}$ from $400 \mathrm{~nm}$ to $800 \mathrm{~nm}$. The measurements are made using a supercontinuum laser source and a set of interference filters with a 10-nm full width at half maximum. The results are shown in Fig. 2(b) (markers), which is fairly well described by Eq. (34) (see the magenta curve). It is noteworthy that we obtain $\xi>0.97$ over the full visible domain and the calculated purity remains actually very high in the near-infrared domain [see the inset of Fig. 2(b)].

\section{Geometric phase identification}

Next the expected geometric phase shaping resulting from spatial rotations of the optical axis is experimentally retrieved by measuring the phase dependence of the contracircularly polarized field component [see Eq. (6)] as a function of the angle $\psi$. This is done by noting that, when $\xi \neq 1$, the azimuth angle $\Psi$ of the reflected polarization ellipse satisfies the relationship $\Psi=\sigma \Phi_{g} / 2$. Therefore, polarimetric analysis of the reflected light as a function of $\psi$ provides quantitative access to the geometric phase. The experiment is performed at $532 \mathrm{~nm}$ by choosing an oil optimizing the condition $\left|r_{\|}+r_{\perp}\right|=\left|r_{\|}-r_{\perp}\right|$, which eases the measurement since we thus deal with a linear polarization state (note that such a condition refers to $\xi=1 / 2$ ). The results are reported in Fig. 3, where the markers refers to the data accumulated over six full rotations of the sample and the solid line refers to
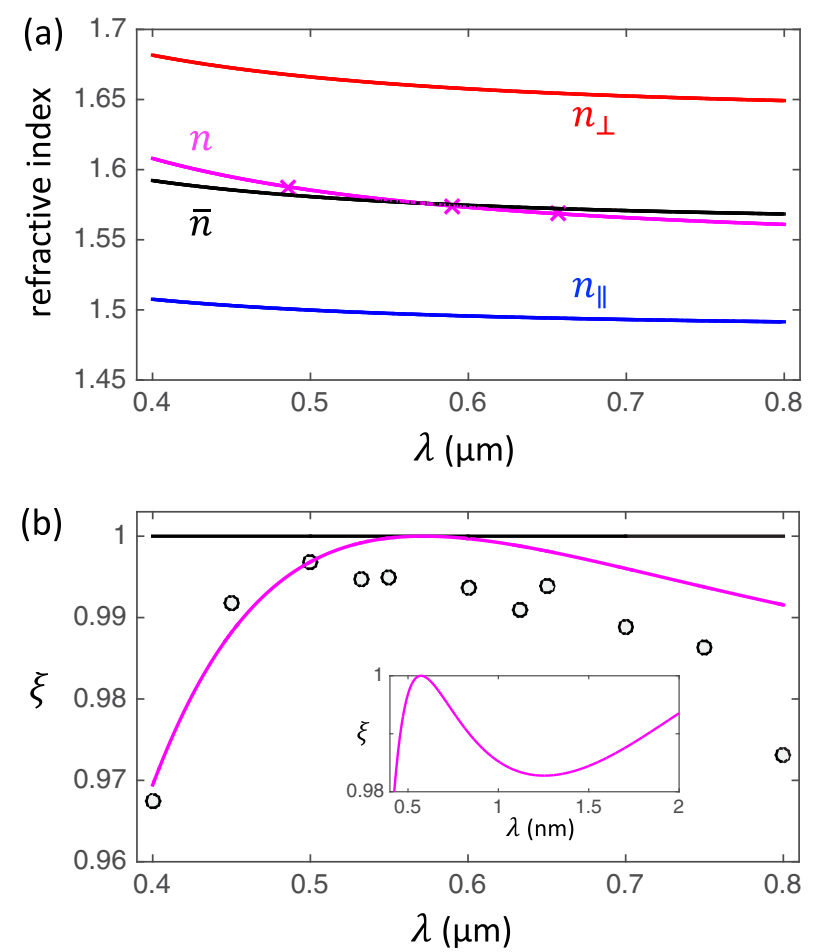

FIG. 2. (a) Dispersion of the refractive indices $n_{\|}$(blue curve), $n_{\perp}$ (red curve), and $\bar{n}$ (black curve) for the calcite slab in the wavelength range $400 \mathrm{~nm}<\lambda<800 \mathrm{~nm}$, from Ref. [20]. The dispersion for the oil that gives the maximal value of $\xi$ in Fig. 1 is shown in magenta. (b) Dispersion of the fraction $\xi$ of the reflected light exhibiting spin angular momentum reversal. The markers show the experimental data. The magenta curve shows the theoretical behavior from Eq. (4) and the black curve the ideally pure behavior $\xi=1$ when $n(\lambda)=\bar{n}(\lambda)$. The inset shows the calculated value up to $\lambda=$ $2 \mu \mathrm{m}$ using the Cauchy extrapolation for the oil and the dispersion equations from Ref. [20].

the expected relationship between the geometric phase and the optical axis orientation angle $\sigma \Phi_{g}=2 \psi$. We conclude with satisfying agreement.

\section{Rotational Doppler experiment}

The optomechanical interpretation of the experiment reported in the preceding section is also worth mentioning in

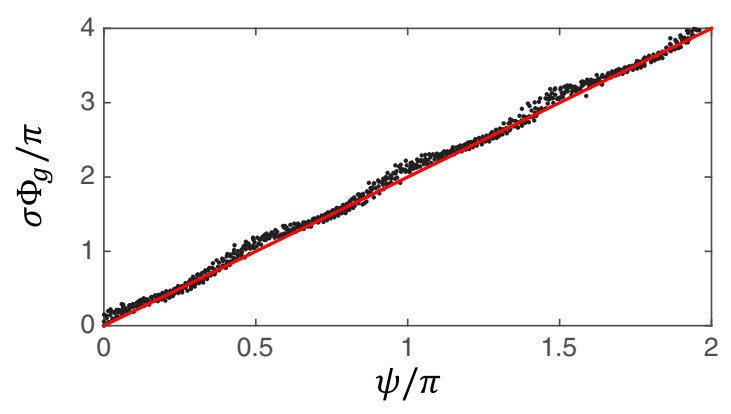

FIG. 3. Product $\sigma \Phi_{g}$ as a function of the optical axis orientation angle $\psi$. The markers show the experimental data and the solid line denotes theory, $\sigma \Phi_{g}=2 \psi$. 
(a)
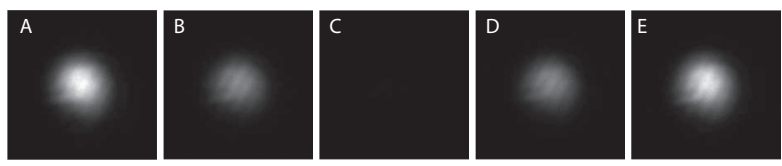

(b)

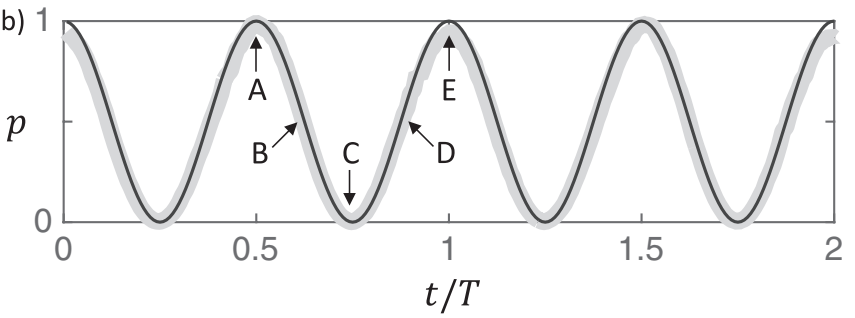

FIG. 4. Rotational Doppler experiment at a 532-nm wavelength as described in the text, where the sample is rotated at constant angular frequency $\Omega$ and the reflected light is recorded after a linear polarizer. (a) Snapshot collection of the optical intensity distribution over one oscillation period of duration $\pi / \Omega$. (b) Normalized timedependent power $p(t)$ as a function of the normalized time $t / T$, with $T=2 \pi / \Omega$. The thick gray curve denotes the experimental data and the thin black curve theory, $p(t)=\cos ^{2}(\Omega t)$.

the context of geometric phase universality regarding time and space, as discussed in Ref. [21]. Indeed, the contribution to the reflected light that reversed its spin angular momentum deposits into the crystal a net $2 \sigma \hbar$ spin angular momentum per photon along the $z$ axis, while the other part does not. Therefore, imparting a constant angular rotation rate to the crystal, $\psi(t)=\Omega t$, a mechanical work is produced by the crystal on the spin-flipped reflected photons, which shifts their frequency. Quantitatively, calling $\omega$ the incident angular frequency, $\delta \omega$ its change, $P$ the power of the reflected light beam component having reversed spin angular momentum contribution, and $\Gamma=2 \sigma P / \omega$ the optical torque exerted on the crystal, the mechanical power balance $\Gamma_{\text {opt }} \Omega+P \delta \omega / \omega=$ 0 leads to $\delta \omega=-2 \sigma \Omega$. This corresponds to the so-called rotational Doppler effect [22,23]. In contrast, the reflected photons having preserved spin angular momentum contribution do not experience any mechanical work and their frequency is thus kept constant. The ensuing temporal beatings between the two orthogonally polarized fields with different frequencies $\omega$ and $\omega+\delta \omega$ thus lead to the rotation of the polarization ellipse of the total field at the angular rate $\Omega$. These beatings can be easily visualized with the naked eye by placing a linear polarizer in the course of the reflected light [see Fig. 4(a)]. The corresponding time-dependent variation of the recorded normalized optical power $p(t)$ is reported in Fig. 4(b) and follows the expected behavior $p(t)=\cos ^{2}(\Omega t)$.

\section{CONCLUDING REMARKS}

Finally, it may be argued that the present strategy relying on the anisotropic Fresnel reflections is associated with a practical drawback, namely, a modest value of the reflected power. Indeed, from the expression of the reflectance under the condition $n=\bar{n}$, namely,

$$
R^{*}=\left|r^{*}\right|^{2}=\frac{\langle n\rangle-\bar{n}}{\langle n\rangle+\bar{n}},
$$

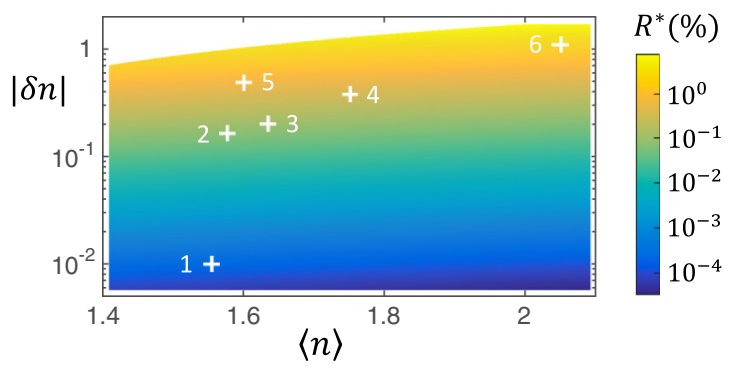

FIG. 5. Map of the reflectance $R^{*}$ in the plane of parameters $(\langle n\rangle, \delta n)$, where $\langle n\rangle=\left(n_{\|}+n_{\perp}\right) / 2$. The plus markers refer to the following practical cases: quartz at $\lambda=\lambda_{\mathrm{D}}(1)$, calcite at $\lambda=\lambda_{\mathrm{D}}$ (2), common nematic liquid crystal E7 at $\lambda=\lambda_{\mathrm{D}}$ (3), very high birefringence nematic liquid crystal at $\lambda=598 \mathrm{~nm}$ [24] (4), diamond subwavelength grating patterns at $\lambda=1550 \mathrm{~nm}$ with pitch $\Lambda=\lambda / 4$ (5), and amorphous silicon subwavelength grating patterns at $\lambda=$ $1550 \mathrm{~nm}$ with pitch $\Lambda=\lambda / 4$ (6). Cases 5 and 6 are evaluated using the second-order effective-medium theory [25].

one gets, in the present case of calcite material, the value $R^{*} \simeq 0.075 \%$ at $\lambda=\lambda_{\mathrm{D}}$. However, much larger values can be obtained by using highly birefringent media having large average refractive index. For instance, subwavelength structuring of amorphous silicon can provide a reflectance value that is much larger than that of calcite, $R^{*} \simeq 2.5 \%$. The trend for the reflectance value as a function of the used material is highlighted in Fig. 5, where $R^{*}$ is calculated in the plane of parameters $(\langle n\rangle, \delta n)$ and where a few practical examples are identified as plus markers. Therefore, the proposed approach offers an attractive option that could foster the elaboration of highly reflective structured anisotropic metainterfaces for applications where purity over a large spectral range matters more than efficiency.

We also notice that space-variant anisotropic elements are only one of the available options to achieve geometric phase shaping of light based on the spin-orbit interaction of light [26], which refers to the coupling between the polarization (spin) degrees of freedom and the spatial (orbital) degrees of freedom. Indeed, isotropic optical systems may also provide geometric phase shaping based on spatial deformations of the wave-vector vectorial spectrum. Specifically, many situations have been unveiled to provide the generation of optical vortex beams (i.e., beams carrying optical phase singularities) with polarization-dependent topological charge $\ell=2 \sigma$ from circularly polarized fields. For instance, this can be done by exploiting either the usual reflection from a conical mirror or the total internal reflection from a dielectric cone as discussed theoretically in Ref. [27] and later demonstrated experimentally in Ref. [28] (mirror reflection) and Ref. [29] (total internal reflection). The use of axisymmetric Fresnel rhomb retarders has also been proposed and experimentally implemented [30-32]. However, despite all these approaches having a good level of achromatic performances, the fabricated devices so far have been endowed with poor spatial resolution (greater than $1 \mathrm{~mm}$ ). Intriguingly, it has also been predicted that planar interfaces between two lossless dielectrics [33] or between vacuum and near-zero $\epsilon$ materials [34] can also behave as polarization-dependent optical vortex 
generators, however without experimental realizations to date. In this context, the present approach has the advantage of having no restriction in terms of the geometric phase pattern imparted to an incident field. From this point of view, our Fresnel approach is both versatile and technology ready.

[1] R. Bhandari, Polarization of light and topological phases, Phys. Rep. 281, 1 (1997).

[2] M. Gecevicius, M. Ivanov, M. Beresna, A. Matijosius, V. Tamuliene, T. Gertus, A. Cerkauskaite, K. Redeckas, M. Vengris, V. Smilgevicius, and P. G. Kazanksy, Toward the generation of broadband optical vortices: Extending the spectral range of a $q$-plate by polarization-selective filtering, J. Opt. Soc. Am. B 35, 190 (2018).

[3] L. Marrucci, E. Karimi, S. Slussarenko, B. Piccirillo, E. Santamato, E. Nagali, and F. Sciarrino, Spin-to-orbital conversion of the angular momentum of light and its classical and quantum applications, J. Opt. 13, 064001 (2011).

[4] Q. Zhan, Cylindrical vector beams: From mathematical concepts to applications, Adv. Opt. Photon. 1, 1 (2009).

[5] M. Born and E. Wolf, Principles of Optics (Pergamon Press, Oxford, 2005).

[6] H. Kikuta, Y. Ohira, and K. Iwata, Achromatic quarter-wave plates using the dispersion of form birefringence, Appl. Opt. 36, 1566 (1997).

[7] A. Niv, G. Biener, V. Kleiner, and E. Hasman, Polychromatic vectorial vortex formed by geometric phase elements, Opt. Lett. 32, 847 (2007).

[8] O. Absil et al., in Proceedings of the Conference on GroundBased and Airborne Instrumentation for Astronomy VI, Edinburgh, 2016, edited by C. J. Evans, L. Simard, and H. Takami, SPIE Proc. (SPIE, Bellingham, 2016), Vol. 9908, p. 99080Q.

[9] G. Destriau and J. Prouteau, Réalisation d'un quart d'onde quasi achromatique par juxtaposition de deux lames cristallines de même nature, J. Phys. Radium 10, 53 (1949).

[10] S. Pancharatnam, Achromatic combinations of birefringent plates. Part II. An achromatic quarter-wave plate, Proc. Indian Acad. Sci. A 41, 137 (1955).

[11] C. Oh and M. J. Escuti, Achromatic diffraction from polarization gratings with high efficiency, Opt. Lett. 33, 2287 (2008).

[12] Y. Li, J. Kim, and M. J. Escuti, in Proceedings of the Conference on Complex Light and Optical Forces VI, SPIE Proc. (SPIE, Bellingham, 2012), Vol. 8274, p. 827415.

[13] N. V. Tabiryan, S. V. Serak, S. R. Nersisyan, D. E. Roberts, B. Y. Zeldovich, D. M. Steeves, and B. R. Kimball, Broadband waveplate lenses, Opt. Exp. 24, 7091 (2016).

[14] N. Tabirian, H. Xianyu, and E. Serabyn, Proceedings of the 2017 IEEE Aerospace Conference (IEEE, Piscataway, 2017), pp. 1-7.

[15] M. Faryad and A. Lakhtakia, The circular Bragg phenomenon, Adv. Opt. Photon. 6, 225 (2014).

[16] J. Kobashi, H. Yoshida, and M. Ozaki, Planar optics with patterned chiral liquid crystals, Nat. Photon. 10, 389 (2016).

[17] J. Kobashi, H. Yoshida, and M. Ozaki, Polychromatic Optical Vortex Generation from Patterned Cholesteric Liquid Crystals, Phys. Rev. Lett. 116, 253903 (2016).

\section{ACKNOWLEDGMENT}

This study was carried out with financial support from the French National Research Agency (Project No. ANR-15CE30-0018), from the DGA, and from the Conseil Régional d'Aquitaine.

[18] M. Rafayelyan and E. Brasselet, Bragg-Berry mirrors: Reflective broadband $q$-plates, Opt. Lett. 41, 3972 (2016).

[19] M. Rafayelyan, G. Agez, and E. Brasselet, Ultrabroadband gradient-pitch Bragg-Berry mirrors, Phys. Rev. A 96, 043862 (2017).

[20] G. Ghosh, Dispersion-equation coefficients for the refractive index and birefringence of calcite and quartz crystals, Opt. Commun. 163, 95 (1999).

[21] K. Y. Bliokh, Y. Gorodetski, V. Kleiner, and E. Hasman, Coriolis Effect in Optics: Unified Geometric Phase and Spin-Hall Effect, Phys. Rev. Lett. 101, 030404 (2008).

[22] P. J. Allen, A radiation torque experiment, Am. J. Phys. 34, 1185 (1966).

[23] B. A. Garetz, Angular Doppler effect, J. Opt. Soc. Am. 71, 609 (1981).

[24] R. Dabrowski, P. Kula, and J. Herman, High birefringence liquid crystals, Crystals 3, 443 (2013).

[25] A. Emoto, M. Nishi, M. Okada, S. Manabe, S. Matsui, N. Kawatsuki, and H. Ono, Form birefringence in intrinsic birefringent media possessing a subwavelength structure, Appl. Opt. 49, 4355 (2010).

[26] K. Y. Bliokh, F. J. Rodríguez-Fortuño, F. Nori, and A. V. Zayats, Spin-orbit interactions of light, Nat. Photon. 9, 796 (2015).

[27] M. Mansuripur, A. R. Zakharian, and E. M. Wright, Spin and orbital angular momenta of light reflected from a cone, Phys. Rev. A 84, 033813 (2011).

[28] H. Kobayashi, K. Nonaka, and M. Kitano, Helical mode conversion using conical reflector, Opt. Exp. 20, 14064 (2012).

[29] N. Radwell, R. D. Hawley, J. B. Götte, and S. Franke-Arnold, Achromatic vector vortex beams from a glass cone, Nat. Commun. 7, 10564 (2016).

[30] T. Wakayama, K. Komaki, Y. Otani, and T. Yoshizawa, Achromatic axially symmetric wave plate, Opt. Exp. 20, 29260 (2012).

[31] T. Wakayama, O. G. Rodríguez-Herrera, J. S. Tyo, Y. Otani, M. Yonemura, and T. Yoshizawa, Generation of achromatic, uniform-phase, radially polarized beams, Opt. Exp. 22, 3306 (2014)

[32] F. Bouchard, H. Mand, M. Mirhosseini, E. Karimi, and R. W. Boyd, Achromatic orbital angular momentum generator, New J. Phys. 16, 123006 (2014).

[33] M. Yavorsky and E. Brasselet, Polarization and topological charge conversion of exact optical vortex beams at normal incidence on planar dielectric interfaces, Opt. Lett. 37, 3810 (2012).

[34] A. Ciattoni, A. Marini, and C. Rizza, Efficient Vortex Generation in Subwavelength Epsilon-Near-Zero Slabs, Phys. Rev. Lett. 118, 104301 (2017). 